\title{
Die perioperative Handhabung von Antikoagulantien in der elektiven Handchirurgie - eine Literaturübersicht
}

\section{Perioperative Anticoagulation in Elective Hand Surgery: A Literature Review}

\author{
Autoren \\ C. Ahrens ${ }^{1}$, F. Unglaub ${ }^{1,4}$, B. Hohendorff ${ }^{2}$, L. P. Müller ${ }^{3}$, C. K. Spies \\ Institute \\ ${ }^{1}$ Vulpius Klinik, Abteilung für Handchirurgie, Bad Rappenau \\ ${ }^{2}$ Abteilung für Hand-, Ästhetische und Plastische Chirurgie, Elbe Klinikum Stade, Stade \\ ${ }^{3}$ Klinik und Poliklinik für Orthopädie und Unfallchirurgie, Universitätsklinik Köln, Köln \\ ${ }^{4}$ Medizinische Fakultät Mannheim der Universität Heidelberg
}

Schlüsselwörter

- Antikoagulantien

- elektive Handchirurgie

Key words

- anticoagulants

- elective hand surgery eingereicht 21.7.2015

akzeptiert $\quad 6.3 .2016$

Bibliografie

DOI http://dx.doi.org/

10.1055/s-0042-104504

Online-Publikation: 23.5.2016

Handchir Mikrochir Plast Chir

2016; 48: 127-135

(c) Georg Thieme Verlag KG

Stuttgart $\cdot$ New York

ISSN 0722-1819

Korrespondenzadresse

Dr. med. Christian K. Spies

Vulpius Klinik

Abteilung für Handchirurgie

Vulpius Straße 29

74906 Bad Rappenau

christianspies27@gmail.com

\section{Zusammenfassung}

$\nabla$

Die vorliegende Arbeit beschreibt auf der Grundlage einer aktuellen Literaturrecherche mögliche Handlungspfade für die perioperative Handhabung von Antikoagulantien in der elektiven Handchirurgie.

\section{Einleitung}

$\checkmark$

Bei Patienten unter Antikoagulation gilt es bei einem anstehenden elektiven chirurgischen Eingriff unter Berücksichtigung des Thromboembolierisikos einerseits und des Blutungsrisikos andererseits abzuwägen, ob die Antikoagulation einfach ausgesetzt werden kann, eine überbrückende Antikoagulation mit Heparin erforderlich ist oder die Antikoagulation in therapeutisch wirksamer Dosierung weitergeführt werden kann/muss. Zweifelsohne ist das Risiko sowohl einer Thromboembolie als auch für eine revisionsbedürftige Blutung bei einem elektiven handchirurgischen Eingriff ein geringeres als z. B. bei der Implantation einer Hüft- oder Kniegelenksprothese. Bis dato gibt es nur wenige Veröffentlichungen und keine Leitlinie zum perioperativen Management von Patienten unter antikoagulatorischer Medikation in der elektiven Handchirurgie. Das Ziel dieses Artikels ist die Präsentation einer aktuellen Literaturübersicht und möglicher Handlungspfade für die perioperative Handhabung von Antikoagulantien in der elektiven Handchirurgie. Das hier skizzierte Vorgehen beruht einerseits auf theoretischen Überlegungen basierend auf den pharmakokinetischen Eigenschaften der gebräuchlichen Antikoagulantien, andererseits auf unserer Erfahrung bezüglich des allgemeinen Blutungsrisikos diverser handchirurgischer Eingriffe. Entsprechend sind die hier vorgestellten Handlungspfade als Vorschläge zu verstehen, die durch zukünftige Studien zu

\section{Abstract}

$\nabla$

This review highlights pathways regarding the handling of anticoagulation in elective hand surgery based on current literature.

prüfen sind. Letztlich ist die Entscheidung bzgl. des Umgangs mit einer antikoagulatorischen Therapie im Rahmen eines elektiven Eingriffes stets individuell zu treffen.

\section{Studienlage zur elektiven Handchirurgie unter Antikoagulation $\nabla$}

Edmunds und Avakian beobachteten bei 121 handchirurgischen Eingriffen unter Antikoagulation mit Phenprocoumon (Marcumar), Clopidogrel (CLO) und Azetylsalizylsäure (ASS) lediglich eine schwere intraoperative Blutung. Bei Marcumareinnahme lag der INR-Wert (International Normalized Ratio) jeweils $\leq 3$. Die Eingriffe umfassten u.a. offene Karpaldachspaltungen, Dupuytren-Operationen mit und ohne Hauttransplantationen, Trapezektomien mit Ligamentrekonstruktion bzw. Sehneninterposition, offene Repositionen und interne Fixationen von distalen Radiusfrakturen, Entfernungen von Mukoidzysten, Ringbandspaltungen, Handgelenksarthroskopien, Transpositionen und Dekompressionen des Nervus ulnaris [1].

Bogunovic und Mitarb. untersuchten in einer prospektiven Kohortenstudie an jeweils $107 \mathrm{~Pa}$ tienten ohne und mit fortlaufender Thrombozytenaggregationshemmung klinische Ergebnisse und Komplikationen nach Hand- und Handgelenkseingriffen. Lediglich bei einem Patienten mit Thrombozytenaggregationshemmung kam es nach einer Handgelenksarthrodese zu einem 
revisionsbedürftigen Hämatom. In Punkto Anzahl postoperativer Ekchymosen und nicht-revisionsbedürftiger Hämatome als auch des klinischen Ergebnisses (DASH-Score, 2-Punkte-Diskrimination) fanden sich keine statistisch signifikanten Unterschiede zwischen beiden Gruppen [2].

Jivan und Mitarb. fanden in einer retrospektiven Untersuchung von 48 Patienten mit perioperativ fortgeführter niedrig dosierter ASS-Dauermedikation $(47 \times 75 \mathrm{mg} / \mathrm{d}, 1 \times 150 \mathrm{mg} / \mathrm{d})$ bei offener Karpaltunnelspaltung in Lokalanästhesie und Blutsperre kein erhöhtes Risiko für Blutungen oder andere Komplikationen [3].

Naito und Mitarb. konnten in einer prospektiven Vergleichsstudie bei 24 offenen Karpaldachspaltungen an 21 Patienten unter Fortsetzung der Antikoagulation mit einem Vitamin-K-Antagonisten (VKA) (Fluidine, Préviscan ${ }^{\circledR}$ ) zeigen, dass es zu keinem relevanten Anstieg der Komplikationsrate gekommen war. Lediglich ein subkutanes Hämatom, das keiner weiteren Therapie bedurfte, trat in der Gruppe mit weitergeführter VKA-Einnahme demgegenüber auf; keine Hämatome in der Gruppe mit unterbrochener VKA-Einnahme [4].

In einer retrospektiven Studie beobachteten Smit und Hooper bei 22 Patienten unter Dauereinnahme von Warfarin (VKA) bei INR-Werten $\leq 3$ keine relevanten intra- oder postoperativen Blutungen. Die Operationen - 9 offene Karpaldachspaltungen in Lokalanästhesie und 13 Dupuytren-Operationen in Regionalanästhesie - erfolgten unter stationären Bedingungen [5].

Wallace und Mitarb. schlussfolgerten aus einer retrospektiven Studie an 39 Patienten mit 55 handchirurgischen Eingriffen unter Dauereinnahme von Warfarin (VKA), dass bei INR-Werten zwischen 1,3 und 2,9 kein Absetzen von Warfarin notwendig sei. Trotz Warfarin-Einnahme beobachteten sie lediglich 2 Hämatome postoperativ, die jedoch keiner Revision bedurften und keine Spätfolgen hinterließen. In 75\% handelte es sich bei den Operationen um Weichteileingriffe (Operationen an Sehnen, Denervierungen, Fasziektomien, offene Karpaldachspaltungen, Neurolysen), in 16\% um Knocheneingriffe wie Amputationen, Trapezektomien und Frakturversorgungen sowie in 9\% um Eingriffe ausschließlich an der Haut (Hauttransplantationen, Z-Plastiken) [6]. Lindsley berichtete in seinem Übersichtsartikel zum perioperativen Management systemischer oraler Antikoagulantien bei handchirurgischen Patienten im Rahmen ambulanter Operationen, dass die Häufigkeit von Blutungskomplikationen in der elektiven Handchirurgie unter Antikoagulation mit Kumarinderivaten (VKA) vergleichbar ist mit der von anderen kleineren chirurgischen Eingriffen, wie Gelenkpunktionen, diagnostischen gastrointestinalen Endoskopien, Routine-Zahneingriffen [7].

Bogunovic und Mitarb. untersuchten in einer prospektiven Kohortenstudie 50 handchirurgische Eingriffe ohne und mit fortlaufender Einnahme von Warfarin (VKA). Die Verteilung von Knochen- gegenüber Weichteileingriffen betrug 1:3. Lediglich bei einem Patienten in der Warfarin-Gruppe kam es 4 Tage nach Entfernung der körpernahen Handwurzelreihe (PRC) zu einem revisionsbedürftigen Hämatom und akutem Karpaltunnelsyndrom. Ekchymosen und nicht-revisionsbedürftige Hämatome waren 2 Wochen postoperativ häufiger in der Warfarin-Gruppe. Nach 4 Wochen zeigten sich diesbezüglich sowie in Bezug auf Schwellung und 2-Punkte-Diskrimination keine statistisch signifikanten Unterschiede zwischen beiden Gruppen [8].
Tab. 1 Risikoabschätzung thromboembolischer Komplikationen.

\begin{tabular}{llll}
\multicolumn{4}{c}{ Risikoabschätzung thromboembolischer Komplikationen } \\
Risiko & Niedrig<5\% & Mittel 5-10\% & Hoch $>\mathbf{1 0 \%}$ \\
$\begin{array}{l}\text { Vorhof- } \\
\text { flimmern }\end{array}$ & CHADS2-Score & CHADS2-Score & CHADS2-Score 5-6 \\
& $0-2$ & $3-4$ & $\begin{array}{l}\text { Zerebrale Ischämie } \\
<3 \text { Monate }\end{array}$ \\
VTE & Einmalige & VTE 3-12 & Akute VTE<3 Monate \\
& VTE $>12$ Monate & Monate & \\
& Keine weiteren & Rezidivierende & Schwere \\
& Risikofaktoren & TVT & Thrombophiliedefekte \\
\hline
\end{tabular}

CHADS2-Score: klinisches Rating zur Abschätzung des Embolierisikos bei Vorhofflimmern; TVT: tiefe Venenthrombose; VTE: venöse Thromboembolie

\section{Perioperative Risikoeinschätzung für thromboembo- lische Ereignisse \\ $\nabla$}

Das Risiko thromboembolischer Komplikationen ist abhängig von der Grunderkrankung, welche die Indikation für die Langzeitantikoagulation darstellt, und dem operativen Eingriff (॰ Tab. 1) [9-11].

Zur Abschätzung des Embolierisikos bei Vorhofflimmern wird in den Leitlinien der $\mathrm{CHADS}_{2}$-Score empfohlen [12]. Der $\mathrm{CHADS}_{2}$-Score ist ein Score-System zur Schlaganfall-Risikoabschätzung bei Vorhofflimmern. Er dient zur Klärung der Indikation für eine antithrombotische Therapie anhand individueller Risikofaktoren. Die Buchstaben CHADS stehen für die einzelnen Risikofaktoren: $\mathrm{C}=$ chronische Herzinsuffizienz, $\mathrm{H}=$ Hypertonie, $\mathrm{A}=$ Alter über 75 Jahre, $\mathrm{D}=$ Diabetes mellitus, $\mathrm{S}=$ Schlaganfall mit 2-facher Wertigkeit. Fehlen beim Vorhofflimmern zusätzliche Risikofaktoren ( $\mathrm{CHADS}_{2}$-Score von 0 ), beträgt das Risiko für embolische Ereignisse 1-2\% jährlich. Patienten mit einem $\mathrm{CHADS}_{2}$-Score von 6 unterliegen einer adjustierten Schlaganfallhäufigkeit von fast $20 \%$ [9,11-13].

Das Risiko thromboembolischer Komplikationen nach venöser Thromboembolie (VTE) ist innerhalb der ersten 3 Monate nach dem Ereignis oder bei Vorliegen von Thrombophiliedefekten hoch ( > 10\% jährlich). Liegt das Ereignis länger zurück oder war der Auslöser der VTE ein operativer Eingriff, ist das Risiko für ein erneutes thromboembolisches Ereignis $<5 \%$ jährlich $[9,11]$ ( $\bullet$ Tab. 1). Neben den Grunderkrankungen, die das thromboembolische Risiko erhöhen, muss das operative thromboembolische Risiko berücksichtigt werden. Abhängig vom Ausmaß der Operation kann durch Aktivierung der Gerinnungskaskade das Risiko für ein thromboembolisches Ereignis vor allem bei großen Verletzungen auf das 10-fache steigen $[9,11]$ ( $\bullet$ Tab. 1).

Die häufigsten Gründe für eine langfristige Antikoagulation mit VKA sind das Vorhofflimmern, der mechanische Herzklappenersatz und die venöse Thromboembolie. Die überbrückende Antikoagulation (Bridging) bei größeren chirurgischen Eingriffen ist wegen des Thromboembolierisikos bei Aussetzen der Antikoagulation notwendig. Dieses Risiko ist nicht nur bei verschiedenen Krankheiten unterschiedlich hoch, sondern wird zusätzlich interindividuell geprägt [14]. Krankheitsbilder, die eine orale Antikoagulation (OAK) erfordern, lassen sich anhand des zu erwartenden Thromboembolierisikos in solche mit hohem $>10 \%$ pro Jahr ohne OAK), mittlerem (4-10\% pro Jahr ohne OAK) und niedrigem Risiko (<4\% pro Jahr ohne OAK) unterteilen. Weitere Faktoren können das Risiko beeinflussen. So erhöht bspw. beim Vorhofflimmern eine gleichzeitig bestehende Herzinsuffizienz 
das Thromboembolierisiko; gleiches gilt für den mechanischen Klappenersatz mit zusätzlichem Vorhofflimmern [14].

Folgende Aufstellung fasst die klinische Risikoeinschätzung für thromboembolische Ereignisse zusammen [14].

Hohes Thromboembolierisiko (circa 10\% pro Jahr und mehr ohne Antikoagulation)

- Tiefe Beinvenenthrombose oder Lungenembolie im zurückliegenden Monat

- Künstliche Herzklappen

- Arterielle Embolie im zurückliegenden Monat

- Vorhofflimmern mit Z.n. ischämischem Ereignis, schwerer Herzinsuffizienz mit eingeschränkter linksventrikulärer Ejektionsfraktion

- Vorhofflimmern mit Thrombus oder dichten Spontanechos im linken Vorhof oder Vorhofohr als Zeichen einer Flussverlangsamung mit Erhöhung von Koagulabilität und damit Echokontrast in der transösophagealen oder transthorakalen Echokardiographie

Mittleres Thromboembolierisiko (circa $4-10 \%$ pro Jahr ohne Antikoagulation)

- Idiopathische tiefe Beinvenenthrombose oder Lungenembolie in den letzten 12 Monaten

- Vorhofflimmern mit begleitendem Diabetes mellitus, arterieller Hypertonie oder höherem Lebensalter

- Bioprothesen (erste 3 Monate)

Niedriges Thromboembolierisiko (unter $4 \%$ pro Jahr ohne Antikoagulation)

- Sekundäre tiefe Beinvenenthrombose oder Lungenembolie in den letzten 12 Monaten

- Idiopathisches Vorhofflimmern

- Bioprothesen (nach 3 Monaten)

Bei Erkrankungen mit niedrigem Thromboembolierisiko ist bei kurzfristig pausierter OAK keine überbrückende Antikoagulation nötig. Hier ist eine dem operativen Eingriff angemessene Standard-Thromboseprophylaxe ausreichend [14].

In der Handchirurgie gelten die allgemeinen Empfehlungen für chirurgische Eingriffe

Der prothrombotische Effekt einer Operation mit der letalen Gefahr von Thrombosen und Embolien sollte beachtet werden. Außerdem erzeugen das Absetzen wie die Wiederaufnahme der Antikoagulation mit VKA eine temporäre hyperkoagulabile Situation, die durch die unterschiedliche Halbwertszeit der Vitamin-K-abhängigen Gerinnungsfaktoren und Gerinnungsinhibitoren erklärt werden kann $[9,11]$.

\section{Perioperative Riskoeinschätzung für Blutungsereig- nisse}

Etwa 3\% aller chirurgischen Eingriffe gehen mit intra- oder postoperativen Blutungen und 0,1\% mit tödlichen Blutungskomplikationen einher [15].

Das für die Aufklärung und Operationsvorbereitung entscheidende individuelle Blutungsrisiko eines Patienten wird durch viele Faktoren modifiziert, darunter patientenspezifische Faktoren wie Alter und Begleiterkrankungen, angeborene oder erworbene Hämostasestörungen, frühere perioperative Blutungen, die perioperative Medikation (Thromboseprophylaxe, andere Anti-
Tab. 2 Fragen zur präoperativen Identifizierung von Patienten mit erhöhtem Blutungsrisiko.

\section{Fragen zur präoperativen Identifizierung von Patienten mit erhöhtem Blutungsrisiko}

1. Haben Sie bei sich selbst vermehrt Nasenbluten ohne erkennbaren Grund festgestellt?

2. Bekommen Sie leicht „blaue Flecken“, ohne sich anzustoßen?

3. Haben Sie bei sich selbst Zahnfleischbluten ohne erkennbaren Grund festgestellt?

4. Treten Blutungen oder blaue Flecken mehr als 1- bis 2-mal pro Woche auf?

5. Haben Sie den Eindruck, bei Schnitt- oder Schürfwunden (z. B. Rasieren) länger nachzubluten?

6. Trat bei Ihnen bereits einmal eine verlängerte oder verstärkte Nachblutung nach oder während Operationen auf (z. B. Mandeloperation, Blinddarmoperation, Geburten)?

7. Trat bei Ihnen eine längere und verstärkte Nachblutung nach dem Ziehen von Zähnen auf?

8. Wurden Ihnen bei einer Operation bereits einmal Blutkonserven oder Blutprodukte gegeben?

9. Gab oder gibt es in der Familie Fälle von vermehrter Blutungsneigung?

10. Nehmen Sie Schmerz- oder Rheumamittel ein?

11. Nehmen Sie weitere Medikamente ein?

12. Für Frauen bzw. Mädchen: Haben Sie den Eindruck, dass Ihre Monatsblutung verlängert (> 7 Tage) oder verstärkt ist?

koagulantien, Thrombozytenaggregationshemmer, nichtsteroidale Antirheumatika (NSAR)), Art und Ausmaß des Eingriffs, Komplexität des Operationsgebietes, Möglichkeiten der Blutstillung, Dringlichkeit des Eingriffs und Erfahrung des Operateurs $[14,15]$. Diese Faktoren können größtenteils nicht quantifiziert werden, weshalb das individuelle Blutungsrisiko letztlich unklar bleibt.

Ziel einer präoperativen Risikoeinschätzung ist es, Patienten mit besonders stark erhöhtem Blutungsrisiko frühzeitig zu identifizieren [15]. Die am besten geeignete Methode zur Identifizierung blutungsgefährdeter Patienten ist die Erhebung der Blutungsanamnese mittels standardisierter Fragebögen. In $\odot$ Tab. 2 ist ein Fragebogen wiedergegeben, der in der präoperativen Diagnostik validiert wurde [15,16]. Bei Patienten mit positiver Anamnese sollte deshalb - insbesondere vor Elektiv- und Hochrisikoeingriffen - eine vollständige hämostaseologische Abklärung erfolgen, möglichst als Stufendiagnostik. Kann diese wegen eines dringenden Eingriffs nicht abgewartet werden, sollte sie nach Möglichkeit trotzdem veranlasst werden, damit die Ergebnisse im weiteren Verlauf in das klinische Management einbezogen werden können [15]. Unabhängige Vorhersageparameter einer periinterventionellen Blutung sind eine positive Blutungsanamnese, mechanische Mitralklappen, ein Heparin-Bridging in einem Zeitfenster von weniger als 24 Stunden nach der Intervention sowie eine aktive Krebserkrankung [17].

Interventionen lassen sich grob unterteilen in solche mit ,hohem" und „nicht hohem“ Blutungsrisiko [18-20]. Im Allgemeinen ist das Blutungsrisiko bei kleineren Eingriffen gering und steigt bei großen Eingriffen. Das Blutungsrisiko handchirurgischer Eingriffe ist stark unterschiedlich. „Die Handchirurgie“ ist somit nicht pauschal einzuordnen, vielmehr bedarf es einer differenzierten Beurteilung des Blutungsrisikos handchirurgischer Eingriffe. 
Eigene Abschätzung des Blutungsrisikos

handchirurgischer Eingriffe

Hohes Risiko:

- Replantationen von Finger, Hand

- Gefäßrekonstuktionen

- Tumorresektionen an Unterarm oder Handgelenk (z. B. palmares Handgelenksganglion)

- Großflächige Debridements

- Großflächige Weichteilrekonstruktionen

- Resektionen einer Skaphoidpseudarthrose mit Interposition eines kortikospongiösen Beckenkammspans

- Korrekturosteotomien des Handgelenkes

- Resektions-Interpositions-Arthroplastiken des Handgelenkes (z.B. PRC)

- Implantationen von Endoprothesen des Handgelenkes

- Eingriffe bei M. Dupuytren

- Vollversteifungen des Handgelenkes

- Mediokarpale Teilarthrodesen

- Offene Repositionen und interne Fixationen (ORIF) des Handgelenkes

- Längerstreckige offene Nervendekompressionen

- Längerstreckige Eingriffe an Sehnen

Niedriges Risiko:

- Resektions-Interpositions-Arthroplastiken der Finger- und Daumengelenke (z. B. Rhizarthrose)

- Kurzstreckige offene Nervendekompressionen

(z.B. Karpaldachspaltung)

- Kurzstreckige Eingriffe an Sehnen

- Arthroplastiken und Arthrodesen der Fingergelenke

- Offene Repositionen und interne Fixationen (ORIF) der Fingergelenke

- Kleine Weichteileingriffe (Mukoidzyste u.a.)

- Ringbandspaltungen

- Arthroskopien

- Geschlossene Repositionen und interne Fixationen von Frakturen an Mittelhand und Finger/Daumen (CRIF)

Bei unklarer Zuordnung in die Risikogruppe mit niedrigem oder hohem Blutungsrisiko empfiehlt sich die zusätzliche Evaluation von möglichen Faktoren, die das Blutungsrisiko erhöhen:

- Positive Blutungsanamnese ( $\bullet$ Tab. 2)

- Medikamenten-Interaktionen

\section{Perioperatives Vorgehen bei Langzeiteinnahme von Thrombozytenaggregationshemmern (TAH) \\ $\nabla$}

Aspirin (ASS) hat eine kurze Plasmahalbwertszeit, hemmt die Zyklooxygenase aber irreversibel, sodass die tatsächliche Wirkdauer vor allem von der Neubildung der Thrombozyten abhängt. Bei normaler Knochenmarksfunktion werden etwa $10 \%$ der Thrombozyten pro Tag ersetzt, sodass 5-6 Tage nach Absetzen von ASS 50\% der Thrombozyten wieder eine normale Funktion aufweisen. Clopidogrel (CLO) als Thienopyridin-Derivat inhibiert die ADP-abhängige Thrombozytenaggregation. Es interagiert weit über die Dauer seiner Plasmahalbwertszeit hinaus mit dem ADP-Rezeptor, sodass eine Rekonstitution von 50\% der ADP-abhängigen Aggregation erst nach etwa 7 Tagen erreicht wird [15].

Prasugrel ist wie Clopidogrel ein Thienopyridin-Derivat und hemmt die ADP-Bindung am P2Y12-Rezeptor der Thrombozyten, was zu einer Hemmung der Plättchenaggregation führt. Es handelt sich um eine irreversible Hemmung. Nach der Verabrei- chung dauert die Plättchenhemmung noch etwa eine Woche an, auch wenn kein Prasugrel mehr verabreicht wird [21].

Anders als ASS hemmen die übrigen NSAR die Thrombozytenaggregation nur reversibel, diese normalisiert sich innerhalb von 1-3 Tagen [15,22]. Selektive Cyclooxygenase-2- (COX-2-) Inhibitoren haben keinen Effekt auf die Thrombozytenaggregation und müssen deshalb perioperativ nicht abgesetzt werden [23].

Ein vorzeitiges Absetzen einer antiaggregatorischen Kombinationstherapie ist bei Patienten nach koronarer Stentimplantation mit einem hohen Risiko kardiovaskulärer Komplikationen assoziiert. Eine perioperative Therapie mit Heparinen bietet keinen ausreichenden Schutz vor Stentthrombosen. Operative Eingriffe sind deshalb, wenn immer möglich, bis zur Beendigung der für die Endothelialisierung des Stents minimal notwendigen Behandlungsdauer zu verschieben [15,24-29]. Wurde die Thrombozytenaggregationshemmung präoperativ unterbrochen, muss diese postoperativ schnellstmöglich wieder aufgenommen werden [23].

Aspirin als Primärprävention - Ziel ist der Erhalt der Gesundheit bzw. die Vorbeugung von Krankheit - sollte maximal 7 Tage vor jeglicher chirurgischer Intervention abgesetzt werden [26]. Die Einnahme von Aspirin als Sekundärprävention - Ziel ist die Verhinderung der Progredienz einer Erkrankung - sollte vor einem chirurgischen Eingriff nicht unterbrochen werden [26]. Falls die Indikation zur Einnahme von Clopidogrel besteht, so sollte diese niemals vor einer nicht kardialen Intervention abgesetzt werden. Clopidogrel muss für mindestens 2-4 Wochen nach einfacher Dilatation, 6 Wochen nach Implantation eines bare metal Stents (BMS) und 12 Monate nach Implantation eines drug-eluting Stents (DES) eingenommen werden. In besonderen Fällen (Hochrisiko-DES, positive Anamnese für Stentthrombose, niedrige Ejektionsfraktion, Diabetes mellitus und Karzinomerkrankung usw.) ist sogar eine Einnahme für ein Jahr und mehr erforderlich [26].

Auch das Anästhesieverfahren hat Einfluss auf das Blutungsrisiko bei einer Operation [30]. Bei Einnahme eines TAH bei gleichzeitiger Heparingabe oder pathologischen Gerinnungsparametern sollte auf ein lokales Anästhesieverfahren verzichtet werden [31]. Bei peripheren regionalanästhesiologischen Verfahren sind nach Möglichkeit Blockaden zu bevorzugen, die kein erhöhtes Risiko für Gefäßpunktionen haben [32]. Nach entsprechender Nutzen-Risiko-Abwägung können sie auch bei Patienten durchgeführt werden, die unter einer Thromboseprophylaxe bzw. einer antithrombotischen Medikation stehen [33].

- Abb. 1 gibt unseren hauseigenen Handlungspfad bei Langzeiteinnahme von TAH im Rahmen elektiver handchirurgischer Eingriffe wieder. Der Handlungspfad basiert auf Empfehlungen der Österreichischen Gesellschaft für Anästhesie, Reanimation und Intensivmedizin für das perioperative Vorgehen bei Patienten unter Therapie mit TAH [34] und lehnt sich an den Algorithmus von Chassot und Mitarb. [26] an.

\section{Perioperatives Vorgehen bei Langzeiteinnahme ora- ler Antikoagulantien \\ $\nabla$}

Bei den oralen Antikoagulantien gilt es zu unterscheiden zwischen Vitamin-K-Antagonisten (VKA) und direkten oralen Antikoagulantien (DOAK). VKA führen indirekt - über die Inhibition der Vitamin-K-abhängigen $\gamma$-Carboxylierung der Gerinnungsfaktoren II, VII, IX und X sowie der gerinnungshemmenden Proteine $\mathrm{C}$ und $\mathrm{S}-$ zu einer Verminderung von aktiven Gerinnungs- 


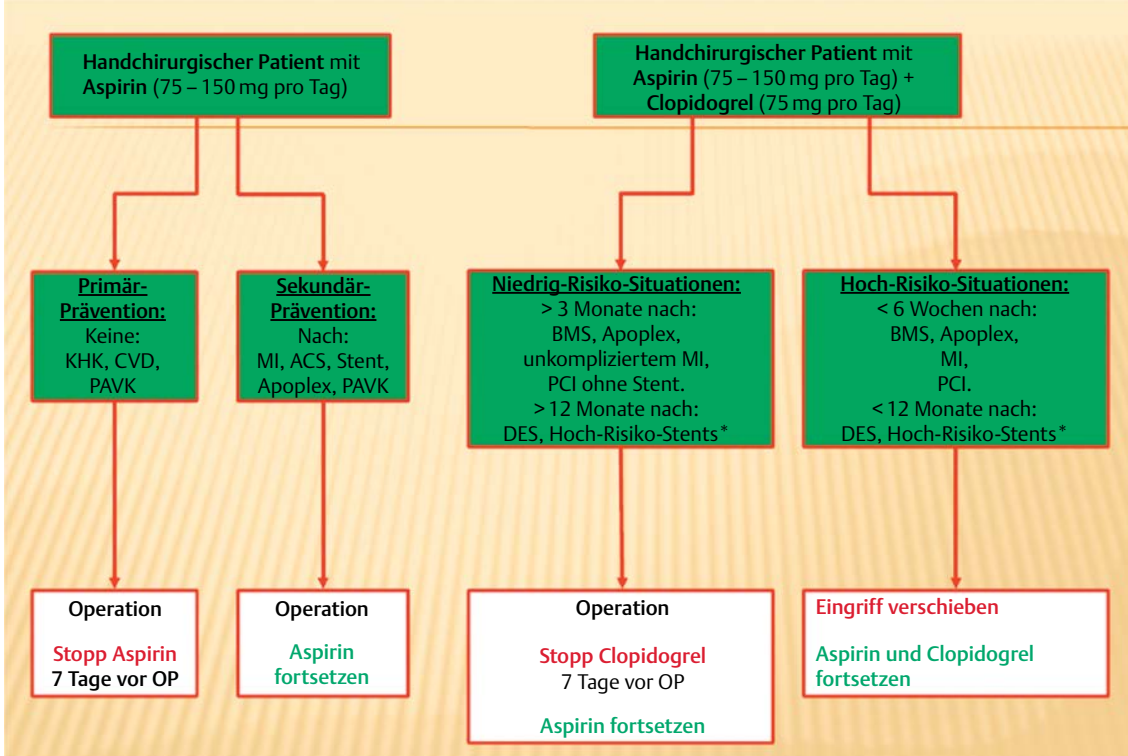

Abb. 1 Handlungspfad an unserer Klinik für das perioperative Vorgehen bei Langzeiteinnahme von Thrombozytenaggregationshemmern in der elektiven Handchirurgie.

ACS: Akutes Koronarsyndrom; BMS: Bare Metal Stent; CVD: Cardiovascular Disease; DES: Drug Eluting Stent; Hoch-Risiko-Stents: lang (>36 mm), proximal, überlappend, multiple Implantationen, Stents bei chronisch totalem Verschluss, in kleinen Gefäßen oder Bifurkationen; KHK: Koronare Herzkrankheit; MI: Myokardinfarkt; PAVK: Peripher-arterielle Verschlusskrankheit; PCI: Perkutane Coronare Intervention.

Nach Absetzen von Aspirin ist die frühzeitige postoperative Wiederaufnahme entscheidend. Primärprävention: Ziel ist der Erhalt der Gesundheit bzw. die Vorbeugung von Krankheit Sekundärprävention: Ziel ist die Verhinderung der Progredienz einer Erkrankung

faktoren. Dagegen hemmen DOAK direkt und reversibel die Gerinnungsfaktoren Xa (Rivaroxaban $\left(\right.$ Xarelto $^{\circledR}$ ) und Apixaban (Eliquis $\left.{ }^{\circledR}\right)$ ) und Ila (Thrombin) (Dabigatran (Pradaxa $\left.{ }^{\circledR}\right)$ ) [35-37]. Der unterschiedliche Wirkmechanismus erklärt den verzögerten Wirkungseintritt der VKA nach 48-72 h gegenüber dem unter DOAK bereits nach $1-3(4) \mathrm{h}$, als auch die unterschiedlich langen Halbwertszeiten (HWZ) von 125-160h unter Phenprocoumon (Marcumar) vs. 5-18h unter den DOAK. Rivaroxaban, das zu 2 Dritteln hepatisch eliminiert wird, besitzt eine HWZ zwischen 5 und $9 \mathrm{~h}$ bei jüngeren Patienten und $9-13 \mathrm{~h}$ bei Patienten $>75$ Jahren $[38,39]$. Apixaban, ebenfalls überwiegend - zu 75\% - hepatisch metabolisiert, weist eine HWZ von etwa $12 \mathrm{~h}$ auf [35,39]. Dabigatran wird dagegen $\mathrm{zu} 80 \%$ unverändert renal ausgeschieden, sodass die HWZ entsprechend von der Kreatinin-Clearance $(\mathrm{KrCl})$ bestimmt wird ( $\geq 80 \mathrm{ml} / \mathrm{min}: 13 \mathrm{~h}, \geq 30 \mathrm{bis}<50 \mathrm{ml} / \mathrm{min}: 16-18 \mathrm{~h}$ ). Bei einer schweren Niereninsuffizienz $(\mathrm{KrCl}<30 \mathrm{ml} / \mathrm{min})$ sollte Dabigatran nicht angewendet werden [40], Rivaroxaban und Apixaban sind bei einer $\mathrm{KrCl}<15 \mathrm{ml} / \mathrm{min}$ kontraindiziert [11]. Besteht aufgrund eines elektiven Eingriffs die Notwendigkeit einer Unterbrechung der oralen Antikoagulation, sollte Marcumar 7-9 Tage präoperativ abgesetzt werden und abhängig vom Thromboembolierisiko eine Überbrückung mit niedermolekularem Heparin (NMH) erfolgen, sofern die $\mathrm{KrCl}>30 \mathrm{ml} / \mathrm{min}$ beträgt. Bei einer $\mathrm{KrCl}$ zwischen 30 und $15 \mathrm{ml} / \mathrm{min}$ kann und bei terminaler Niereninsuffizienz sollte das Bridging mit unfraktioniertem Heparin (UFH) erfolgen [9,11]. Die Gabe von NMH erfolgt, sobald der INR-Wert unterhalb des angestrebten Zielbereichs liegt. Am Morgen der Operation bzw. bei 2-mal täglicher Gabe am vorhergehenden Abend wird Heparin abgesetzt und bei adäquater Hämostase frühestens $6 \mathrm{~h}$ postoperativ oder am ersten postoperativen Tag erneut begonnen. Auch die Wiederaufnahme der VKA-Therapie kann am ersten postoperativen Tag erfolgen, wenn das Blutungsrisiko dies zulässt $[9,11,41]$.

Bei den DOAK ist das präoperative antikoagulationsfreie Intervall abhängig vom eingenommenen oralen Antikoagulans, von der Begleitmedikation, der Nierenfunktion des Patienten und dem Blutungsrisiko des Eingriffes [11,42]. Von Herstellerseite wird ein Pausieren der DOAK von 2-4 Halbwertszeiten (HWZ) präoperativ (1-5 Tage) und die frühzeitige Wiederaufnahme postoperativ empfohlen $[35,37,38,40,43]$.
Unter Berücksichtigung des o.g. Blutungsrisikos bei handchirurgischen Eingriffen sowie Literaturempfehlungen zum perioperativen Management von DOAK sind in $\odot$ Tab. $3 \mathrm{Ab}$ - und Ansetzungsintervalle vor und nach elektiven handchirurgischen Eingriffen in Abhängigkeit von der Nierenfunktion angeführt [11,39, 42,44-47].

Nach der S3-Leitlinie zur Prophylaxe venöser Thromboembolien ist eine medikamentöse Thromboseprophylaxe bei Operationen und Verletzungen an Gelenken, Knochen und Weichteilen von Hand und Unterarm grundsätzlich nicht notwendig [48].

Die Notwendigkeit einer Bridging-Therapie mit Heparinen bei Patienten unter DOAK-Medikation ist fragwürdig, deshalb kann eine Risikobeurteilung bezüglich perioperativer Thromboembolie- und Blutungsrisiken hilfreich sein $[39,41]$. In den Abschnitten „Perioperative Risikoeinschätzung für thromboembolische Ereignisse“ inklusive $\bullet$ Tab. 1 und „Perioperative Riskoeinschätzung für Blutungsereignisse“ sind entsprechende Risikoeinteilungen tabellarisch aufgeführt. Demnach ist die Überbrückung einer bestehenden Antikoagulation mittels Heparin bei hohem Thromboembolierisiko unabhängig vom Blutungsrisiko der vorgesehenen Operation angebracht. Bei mittlerem Thromboembolierisiko ist sie in Kombination mit niedrigem Blutungsrisiko diskutabel, verbunden mit hohem Blutungsrisiko ebenso wie bei niedrigem Thromboembolierisiko unabhängig vom Blutungsrisiko nicht angebracht ( $\bullet$ Tab. 4) [39]. Untermauert wird dies durch eine systemische Übersichtsarbeit zur Sicherheit und Wirksamkeit eines periprozeduralen Bridgings bestehender Antikoagulantien. Die Überbrückung mittels Heparin bei nicht hohem thromboembolischen Risiko im Kontext von Eingriffen mit hohem Blutungsrisiko sollte demnach vermieden werden $[49,50]$.

Von French Study Group on Thrombosis and Haemostasis wird bei chirurgischen Interventionen mit moderatem bis hohem Blutungsrisiko empfohlen, die letzte Gabe der DOAK am 5. Tag präoperativ zu verabreichen (letzte Gabe morgens) und eine Überbrückung mit Heparin ab dem 3. Tag präoperativ durchzuführen [51]. Die Wiederaufnahme der oralen Antikoagulation mit DOAK sollte erst dann erfolgen, wenn das Risiko für Blutungskomplikationen ausreichend gering ist (frühestens 2 Tage postoperativ). Je nach Operation ist ggf. die erste Phase der 


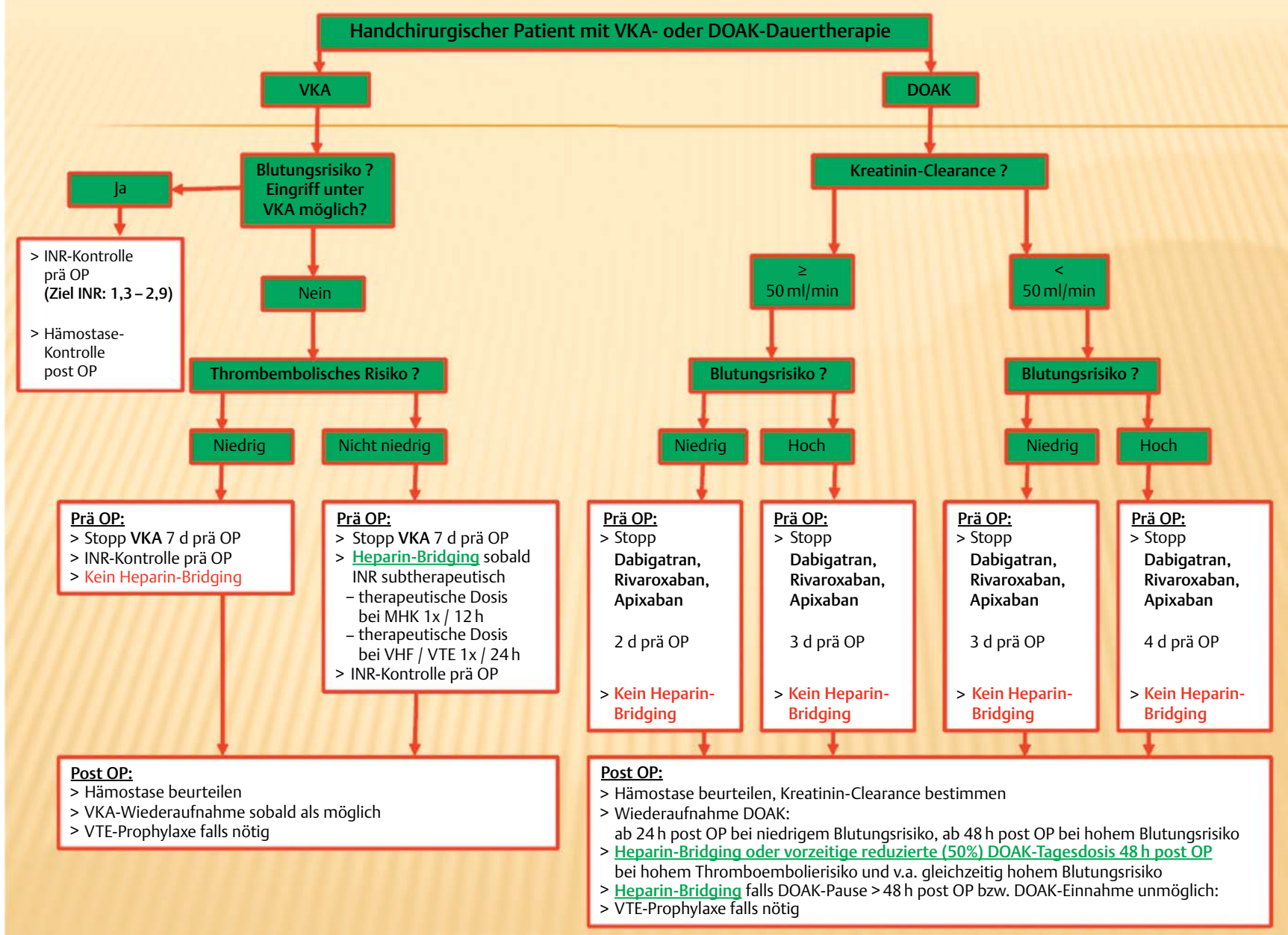

Abb. 2 Möglicher Handlungspfad für das perioperative Vorgehen bei Langzeiteinnahme von Vitamin-K-Antagonisten und direkten oralen Antikoagulantien in der elektiven Handchirurgie, den es durch weitere Studien zu überprüfen gilt.

DOAK: direkte orale Antikoagulantien; VKA: Vitamin-K-Antagonisten; d: Tag(e); h: Stunde(n); INR: International Normalized Ratio; MHK: Mechanische Herzklappe; OP: Operation; VHF: Vorhofflimmern; VTE: venöse Thromboembolie.

CAVE: Heparin-Bridging in Abhängigkeit der Kreatinin-Clearance ( $\mathrm{KrCl})$ :

$\mathrm{KrCl}>30 \mathrm{ml} / \mathrm{min}$ : kann mit niedermolekularem Heparin ( $\mathrm{NMH}$ ) erfolgen

$\mathrm{KrCl}$ zwischen 30 und $15 \mathrm{ml} / \mathrm{min}$ : kann mit unfraktioniertem Heparin (UFH) erfolgen

Terminale Niereninsuffizienz: sollte mit unfraktioniertem Heparin (UFH) erfolgen.

Das VKA-Bridging im Fall der Kombination (nicht niedriges) mittleres Thromboserisiko + hohes Blutungsrisiko erfordert eine individuelle Entscheidung aufgrund der Blutungsgefahr.

Eine gestörte gastrointestinale Absorption oder nicht mögliche orale Medikamenteneinnahme kann ein DOAK-Bridging erforderlich machen.

(ASS) kann perioperativ weitergeführt, Clopidogrel (CLO) sollte rechtzeitig vor Operation abgesetzt werden. Kardiovaskuläre Hochrisiko-Situationen erfordern eine antiaggregatorische Kombinationstherapie mit ASS und CLO und verbieten einen elektiven Eingriff. Eingriffe unter Einnahme von Vitamin-K-Antagonisten (VKA) sind bei niedrigem Blutungsrisiko und INR-Werten (International Normalized Ratio) zwischen 1,3 und 2,9 möglich, ein hohes Blutungsrisiko erfordert ein rechtzeitiges Absetzen der VKA sowie ein Heparin-Bridging bei gleichzeitig erhöhtem Thromboembolierisiko. Direkte orale Antikoagulantien (DOAK) sollten in Abhängigkeit von Kreatinin-Clearance und Blutungsrisiko abgesetzt werden, ein Heparin-Bridging scheint nur in wenigen Ausnahmefällen notwendig.

Interessenkonflikt: Nein.

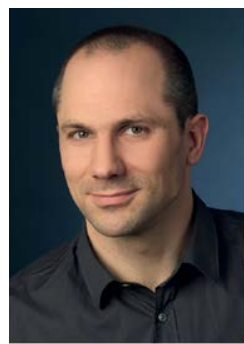

Dr. med. Carsten Ahrens

geb. 12.02.1974 in Rotenburg a.d. Fulda, 2002-2009 Studium der Humanmedizin an der Ruprecht-Karls Universität Heidelberg, 2010 Approbation, 2011 Promotion. Seit 2010 Facharztausbildung Klinik für Orthopädie und Unfallchirurgie, Vulpius Klinik Bad Rappenau (Prof. Dr. med. M. Clarius). Kooperative Rotation Neckar-Odenwald-Kliniken Mosbach und Buchen 2011-2013: Klinik für Allgemein-, Viszeral- und Gefäßchirurgie (Dr. med. H. Kirr), Klinik für Anästhesiologie und Intensivmedizin (PD Dr. med. H. Genzwürker), Klinik für Orthopädie und Unfallchirurgie (Dr. med. B. Gritzbach). Hausinterne Rotation Handchirurgie Vulpius Klinik Bad Rappenau (Prof. Dr. med. P. Hahn und Prof. Dr. med. F. Unglaub) Januar-Juni 2014. 


\section{Literatur}

1 Edmunds I, Avakian Z. Hand surgery on anticoagulated patients: a prospective study of 121 operations. Hand Surg 2010; 15: 109-113

2 Bogunovic L, Gelberman RH, Goldfarb CA et al. The impact of antiplatelet medication on hand and wrist surgery. J Hand Surg Am 2013; 38: 1063-1070

3 Jivan S, Southern S, Majumder S. Re: the effects of aspirin in patients undergoing carpal tunnel decompression. J Hand Surg Eur Vol 2008; 33: 813-814

4 Naito K, Lequint T, Zemirline A et al. Should we stop oral anticoagulants in the surgical treatment of carpal tunnel syndrome? Hand 2012; 7: $267-270$

5 Smit A, Hooper G. Elective hand surgery in patients taking warfarin. J Hand Surg Eur Vol 2004; 29: 204-205

6 Wallace DL, Latimer MD, Belcher HJ. Stopping warfarin therapy is unnecessary for hand surgery. J Hand Surg Eur Vol 2004; 29: 203-205

7 Lindsley $R C$. Perioperative management of systemic oral anticoagulants in patients having outpatient hand surgery. J Hand Surg Am 2008; 33: 1205-1207

8 Bogunovic L, Gelberman RH, Goldfarb CA et al. The Impact of Uninterrupted Warfarin on Hand and Wrist Surgery. J Hand Surg Am 2015; 40: $2133-2140$

9 Schellong SM, Haas S, Siebenlist S. Überbrückung Pausieren und Wechsel von Antikoagulantien in der Unfallchirurgie. Unfallchirurg 2010; 113: 901-907

10 Spyropoulos AC, Douketis JD, Gerotziafas G et al. Periprocedural antithrombotic and bridging therapy: recommendations for standardized reporting in patients with arterial indications for chronic oral anticoagulant therapy. JTH 2012; 10: 692-694

11 Eisele R, Melzer N, Bramlage P. Perioperatives Gerinnungsmanagement bei oraler Antikoagulation. Chirurg 2014; 85: 513-519

12 European Heart Rhythm Association (EHRA), European Association for Cardio-Thoracic Surgery (EACTS).Camm AJ et al. Guidelines for the management of atrial fibrillation: the Task Force for the Management of Atrial Fibrillation of the European Society of Cardiology (ESC). EHJ 2010; 31: 2369-2429

13 Bridge Study Investigators. Bridging anticoagulation: is it needed when warfarin is interrupted around the time of a surgery or procedure? Circulation 2012; 125: e496-e498

14 Bauersachs $R$, Schellong S, Haas $S$ et al. Überbrückung der oralen Antikoagulation bei interventionellen Eingriffen. Dtsch Ärztebl Int 2007; 104: $1237-1244$

15 Tiede A. Perioperatives hämostaseologisches Management. Chirurg 2007; 78: 69-79

16 Koscielny J, von Tempelhoff GF, Ziemer S et al. A practical concept for preoperative management of patients with impaired primary hemostasis. Clin Appl Thromb Hemost 2004; 10: 155-166

17 Tafur AJ, McBane R, Wysokinski WE et al. Predictors of major bleeding in peri-procedural anticoagulation management. JTH 10: 261-267

18 Douketis JD, Johnson JA, Turpie AG. Low-molecular-weight heparin as bridging anticoagulation during interruption of warfarin: assessment of a standardized periprocedural anticoagulation regimen. Arch Int Med 2004; 164: 1319-1326

19 Jafri SM. Periprocedural thromboprophylaxis in patients receiving chronic anticoagulation therapy. Am Heart J 2004; 147: 3-15

20 Spandorfer $J$. The management of anticoagulation before and after procedures. Med Clin North Am 2001; 85: 1109-1116

21 Jernberg T, Payne $C D$, Winters KJ et al. Prasugrel achieves greater inhibition of platelet aggregation and a lower rate of non-responders compared with clopidogrel in aspirin-treated patients with stable coronary artery disease. EHJ 2006; 27: 1166-1173

22 Smith MS, Muir H, Hall R. Perioperative management of drug therapy, clinical considerations. Drugs 1996; 51: 238-259

23 Vogel Kahmann I, Ruppen W, Lurati Buse G et al. Langzeitmedikation und perioperatives Management. Internist 2011; 52: 89-98

24 Biondi-Zoccai GG, Lotrionte M, Agostoni P et al. A systematic review and meta-analysis on the hazards of discontinuing or not adhering to aspirin among 50,279 patients at risk for coronary artery disease. EHJ 2006; 27: 2667-2674

25 Burger W, Chemnitius JM, Kneissl GD et al. Low-dose aspirin for secondary cardiovascular prevention - cardiovascular risks after its perioperative withdrawal versus bleeding risks with its continuation - review and meta-analysis. J Int Med 2005; 257: 399-414
26 Chassot PG, Delabays A, Spahn DR. Perioperative antiplatelet therapy: the case for continuing therapy in patients at risk of myocardial infarction. Br J Anaesth 2007; 99: 316-328

27 Mangano DT. Multicenter Study of Perioperative Ischemia Research Group. Aspirin and mortality from coronary bypass surgery. N Engl J Med 2002; 347: 1309-1317

28 Nuttall GA, Brown MJ, Stombaugh JW et al. Time and cardiac risk of surgery after bare-metal stent percutaneous coronary intervention. Anesthesiology 2008; 109: 588-595

29 Rabbitts JA, Nuttall GA, Brown MJ et al. Cardiac risk of noncardiac surgery after percutaneous coronary intervention with drug-eluting stents. Anesthesiology 2008; 109: 596-604

30 Kozek-Langenecker SA. Lokoregionalanästhesie und Blutgerinnung. Behandlung mit Thrombozytenfunktionshemmern. Anaesthesist 2003; 52: 549-563

31 Lepper W, Kelm M. Perioperative Therapie mit Thrombozytenaggregationshemmern. Anaesthesist 2007; 56: 592-598

32 Abrahams MS, Aziz MF, Fu RF et al. Ultrasound guidance compared with electrical neurostimulation for peripheral nerve block: a systematic review and meta-analysis of randomized controlled trials. $\mathrm{Br} \mathrm{J}$ Anaesth 2009; 102: 408-417

33 Neuburger $M$, Buttner J. Komplikationen bei peripherer Regionalanästhesie. Anaesthesist 2011; 60: 1014-1026

34 Österreichische Gesellschaft für Anästhesie Reanimation und Intensivmedizin. Richtlinien perioperative Gerinnung 2010

35 Bristol-Myers Squibb. Fachinformation Eliquis ${ }^{\circledR} 2012$

36 Kreutz $R$. Pharmacodynamic and pharmacokinetic basics of rivaroxaban. Fundam Clin Pharmacol 2012; 26: 27-32

37 Bauersachs $R$, Gogarten W, Hach-Wunderle V et al. Perioperatives Management der Antikoagulation mit Rivaroxaban - Konsensus einer interdisziplinären Arbeitsgruppe. Klinikarzt 2012; 424-431

38 Bayer Pharma. Fachinformation Xarelto ${ }^{\circledR} 2012$

39 Liew A, Douketis J. Perioperative management of patients who are receiving a novel oral anticoagulant. Intern Emerg Med 2013; 8: 477-484

40 Spannagl M, Bauersachs R, Debus ES et al. Therapie mit Dabigatran. Periinterventionelles Management und Interpretation von Gerinnungstests. Haemostaseologie 2012; 32: 294-305

41 Douketis JD, Spyropoulos AC, Spencer FA et al. Perioperative management of antithrombotic therapy: Antithrombotic Therapy and Prevention of Thrombosis. Chest 2012; 141: e326S-e350S

42 Connolly G, Spyropoulos AC. Practical issues, limitations, and periprocedural management of the NOAC's. J Thromb Thrombolysis 2013; 36: 212-222

43 Boehringer Ingelheim. Fachinformation Pradaxa ${ }^{\circledR} 2014$

44 Schellong SM, Haas S. Perioperative Thromboseprophylaxe - Neue orale Antikoagulantien und ihre Anwendung im Umfeld operativer Eingriffe. Anaesthesiol Intensivmed Notfallmed Schmerzther 2012; 47: $266-272$

45 Spyropoulos AC, Douketis JD. How I treat anticoagulated patients undergoing an elective procedure or surgery. Blood 2012; 120: 29542962

46 Schulman S, Crowther MA. How I treat with anticoagulants in 2012: new and old anticoagulants, and when and how to switch. Blood 2012: 119: 3016-3023

47 Vilchez JA, Gallego P, Lip GY. Safety of new oral anticoagulant drugs: a perspective. Ther Adv Drug Saf 2014; 5: 8-20

48 AWMF. Arbeitsgemeinschaft der Wissenschaftlichen Medizinischen Arbeitsgemeinschaften. S3-Leitlinie zur Prophylaxe venöser Thromboembolien 2009

49 Siegal D, Yudin J, Kaatz $S$ et al. Periprocedural heparin bridging in patients receiving vitamin $\mathrm{K}$ antagonists: systematic review and meta-analysis of bleeding and thromboembolic rates. Circulation 2012; 126: $1630-1639$

50 Gallego P, Apostolakis S, Lip GY. Bridging evidence-based practice and practice-based evidence in periprocedural anticoagulation. Circulation 2012; 126: 1573-1576

51 Sie P, Samama CM, Godier A et al. Surgery and invasive procedures in patients on long-term treatment with direct oral anticoagulants: thrombin or factor-Xa inhibitors. Arch Cardio Dis 2011; 104: 669-676

52 Schlitt A, Jambor C, Spannagl $M$ et al. Perioperativer Umgang mit Antikoagulanzien und Thrombozytenaggregationshemmern. Dtsch Ärztebl Int 2013; 110: 525-532 
53 Lahtela H, Rubboli A, Schlitt A et al. Heparin bridging vs. uninterrupted oral anticoagulation in patients with Atrial Fibrillation undergoing Coronary Artery Stenting. Circ J 2012; 76: 1363-1368

54 Camm AJ, Lip GY, De Caterina R et al. 2012 focused update of the ESC Guidelines for the management of atrial fibrillation: an update of the 2010 ESC Guidelines for the management of atrial fibrillation. EHJ 2012; 33: 2719-2747
55 Healey JS, Eikelboom J, Douketis J et al. Periprocedural bleeding and thromboembolic events with dabigatran compared with warfarin: results from the Randomized Evaluation of Long-Term Anticoagulation Therapy (RE-LY) randomized trial. Circulation 2012; 126: 343-348

56 Wysokinski WE, McBane RD. Periprocedural bridging management of anticoagulation. Circulation 2012; 126: 486-490 